\title{
Reduction in Medical Care Cost Associated with Radiofrequency Catheter Ablation of Accessory Pathways
}

\author{
Michael de Buitleir, MD, Joao Sousa, MD, Steven F. Bolling, MD, Rafel El-Atassi, MD, \\ Hugh Calkins, MD, Jonathan J. Langberg, MD, William H. Kou, MD, and Fred Morady, MD
}

The cost of definitive therapy was compared in 25 patients who underwent radiofrequency catheter ablation of accessory pathways in 1990 and 25 patients who underwent surgical ablation of accessory pathways in 1989. In the radiofrequency group, 23 of 25 patients had a single accessory pathway and the remaining 2 patients each had 2 accessory pathways. In the surgical group, 20 patients had a single accessory pathway and 5 patients each had 2 accessory pathways. The success rate was $96 \%$ in each group. The mean duration of hospitalization was $3 \pm 1$ days in the radiofrequency group and $9 \pm 4$ days in the surgical group $(p<0.0001)$. All the cost data are expressed in fiscal year 1990/1991 dollar values. The total cost of therapy in the radiofrequency group was $\$ 14,919 \pm \$ 6,740$ compared with $\$ 53,265 \pm \$ 12,755$ in the surgical group ( $<<0.0001$ ). The cost of radiofrequency ablation consisted of a hospital charge of $\$ 7,753 \pm \$ 3,472$ and physician fees of $\$ 7,166$ $\pm \$ 3,439$. The hospital charge included charges for use of the electrophysiology laboratory, hospital stay, electrocardiograms, echocardiograms and blood studles. The cost of surgery consisted of a hospital charge of $\$ \mathbf{\$ 7 , 7 0 8} \pm \mathbf{\$ 1 0 , 1 7 9}$ and physician fees of $\$ 15,557 \pm \$ 3,149$. The hospital charge in the surgical group included the costs of a baseline electrophysiology study, inhospital care and a follow-up office visit. In conclusion, radiofrequency catheter ablation of accessory pathways results in a dramatic reduction in the cost of definitive therapy in patients with the Wolff-Parkinson-White syndrome.

(Am J Cardiol 1991;68:1656-1661)

From the Department of Internal Medicine, Division of Cardiology, and the Department of Cardiothoracic Surgery, University of Michigan Medical Center, Ann Arbor, Michigan. Manuscript received June 10, 1991; revised manuscript received August 16, 1991, and accepted August 18.

Address for reprints: Michael de Buitleir, MD, Department of Medicine, Cardiology Section, University of Wisconsin Hospital, H6/344, 600 Highland Avenue, Madison, Wisconsin 53792.

1656 THE AMERICAN JOURNAL OF CARDIOLOGY VOLUME 68
$\mathrm{R}$ adiofrequency catheter ablation has been shown to be a safe and efficacious means of interrupting accessory pathway conduction..$^{1,2}$ This treatment method has a high degree of patient acceptance because it eliminates the need for openchest surgery. An important additional advantage of this therapy is the anticipated cost-savings associated with its use, but few data on this aspect have been published to date. Therefore, this study describes the cost of accessory pathway ablation using radiofrequency current and compares this cost with that of a standard surgical approach. ${ }^{3}$

\section{METHODS}

This was a retrospective study of 25 consecutive patients who underwent surgical ablation of accessory pathways in 1989 and 25 consecutive patients who underwent radiofrequency ablation of accessory pathways in 1990. All the patients in the study were referred to the University of Michigan Medical Center for management of recurrent supraventricular tachyarrhythmias.

Electrophysiologic study: All patients underwent a diagnostic electrophysiologic test at least 5 half-lives after discontinuation of all antiarrhythmic drugs and after providing informed consent. Three $6 \mathrm{Fr}$ quadripolar catheters were inserted into a femoral vein and positioned at the high right atrium, across the tricuspid valve to record the His bundle electrogram and against the right ventricular apex or septum. An additional quadripolar catheter was inserted into the subclavian or internal jugular vein and positioned in the coronary sinus. After the catheters were in place, 3,000 $\mathrm{U}$ of heparin were administered intravenously. Programmed atrial and ventricular stimulation was performed using a Bloom DTU 201 stimulator (Bloom Associates, Narbeth, Pennsylvania). Standard techniques were used to elucidate the presence of an accessory pathway and to confirm its participation as a critical limb of the tachycardia circuit. ${ }^{4}$ A Siemens-Elema Mingograf 7 ink-jet recorder provided both a continuous oscilloscopic display and a permanent record of leads $\mathrm{V}_{1}, \mathrm{I}$ and III and the intracardiac electrograms.

Radiofrequency ablation group: The group comprised 11 men and 14 women (mean age $40 \pm 16$ 
years, range 14 to 74 ). Mean duration of symptoms was $21 \pm 17$ years (range of 1 month to 60 years). They had been treated with a mean of $2 \pm 1$ antiarrhythmic drugs before undergoing radiofrequency ablation and most of these drugs had been poorly tolerated or were ineffective. Twenty-three of the 25 patients had a single accessory pathway and the remaining 2 patients each had 2 accessory pathways. Fifteen of the accessory pathways were left-sided, 9 were septal and 3 were right-sided; 17 displayed manifest preexcitation and 10 were concealed.

On completion of the diagnostic portion of the electrophysiologic study, radiofrequency catheter ablation was attempted during the same session. The ablation protocol was approved by the Human Research Committee at the University of Michigan and all patients provided written informed consent before undergoing the procedure. Radiofrequency ablation was performed using a 7Fr quadripolar deflectable tip catheter with a $4 \mathrm{~mm}$ distal electrode and $5 \mathrm{~mm}$ interelectrode spacing (Mansfield, Watertown, Massachusetts). For left-sided accessory pathways, the catheter was advanced from the femoral artery to the left ventricle and positioned in the atrioventricular sulcus under the mitral valve anulus. For right-sided accessory pathways, the catheter was advanced from the inferior cava to the right atrium at the level of the tricuspid valve. For septal accessory pathways, the ablation catheter was advanced from the superior or inferior vena cava to the coronary sinus os or the anterior atrial septum. A detailed description of the mapping and ablation technique has been previously provided. ${ }^{2}$

On completion of the radiofrequency ablation procedure, the patients were admitted to a telemetry unit for 48 hours of monitoring. Three serial blood samples were obtained at 8-hour intervals for determination of the plasma concentrations of creatine kinase and its MB subfraction. A daily 12-lead electrocardiogram was recorded and cross-sectional echocardiography and Doppler study were performed within 24 hours of the radiofrequency ablation. In patients in whom the accessory pathway was concealed, a brief electrophysiologic test was performed after 48 hours to confirm successful ablation. In patients in whom the accessory pathway was manifest, the absence of delta waves on the 48hour electrocardiogram was regarded as evidence of a successful outcome and a repeat electrophysiologic study was not performed before discharge from the hospital. All patients underwent a final electrophysiologic test after 3 months on an outpatient basis to assess the long-term efficacy of the procedure.

Surgical group: This group comprised 14 men and 11 women (mean age $30 \pm 11$ years, range 13 to 60 ). The mean duration of symptoms was $11 \pm 11$ years (range 1 month to 40 years). The patients had been treated with $2 \pm 2$ antiarrhythmic drugs before undergoing surgery. These drugs had been ineffective or associated with unacceptable side effects for almost all of the patients. Twenty of the 25 patients had a single accessory pathway and the remaining 5 patients each had 2 accessory pathways. Twenty of the accessory pathways were left-sided, 8 were septal and 2 were right-sided; 20 displayed manifest preexcitation and 10 were concealed.

Surgical ablation was performed using an endocardial approach. ${ }^{3}$ After the induction of general anesthesia, the chest was opened through a median sternotomy incision. The accessory pathway(s) was localized using standard mapping techniques ${ }^{4}$ and the patient was then placed on cardiopulmonary bypass. When the surgical ablation was completed the patient was weaned from cardiopulmonary bypass and intraoperative epicardial mapping was repeated to evaluate the efficacy of the surgical dissection. Before discharge from the hospital, bedside pacing using temporary epicardial pacing leads was performed to confirm a successful surgical result.

Cost analysis: Retrospective review of each patient's clinical record was performed to determine the number, duration and dates of hospital admissions and the number and dates of office visits that were related directly to the ablation procedure. Only charges billed from the University of Michigan Medical Center were included in the present analysis. The costs incurred by each patient were divided into hospital and physician charges. Itemized bills were obtained from the patients' accounts office for all ablation-related admissions and office visits. The hospital costs include the charges for room and board, operating room or electrophysiology laboratory charges, and charges for nursing and ancillary care, drugs, blood tests, $x$-rays, electrocardiograms and echocardiograms. The analysis incorporated the cost of a 1month follow-up office visit and an electrocardiogram at rest for all patients. All patients treated surgically had a chest $\mathrm{x}$-ray during the follow-up office visit and this cost was also included.

Physician charges for all ablation-related hospital admissions and office visits were obtained from the relevant physician-associates groups at the University of Michigan Medical Center. The cost of the 3-month follow-up electrophysiologic study in the radiofrequency ablation group was excluded from the present analysis because the indication for this study was based on the investigational nature of the procedure and the need to document accurately its long-term effects. As radiofrequency ablation becomes established in clinical practice, this follow-up study will not be necessary.

To conduct a valid comparison and to provide cost data that are meaningful in 1991, an adjustment was made to convert all costs to their 1990/1991 fiscal year dollar values. The annual increase in hospital charges 


\begin{tabular}{|c|c|c|c|c|c|c|}
\hline Pt. No. & Age $(y r)$ & No. of APs & $\begin{array}{c}\text { Hospital } \\
\text { Stay (days) }\end{array}$ & $\begin{array}{c}\text { Hospital } \\
\text { Charges (\$) }\end{array}$ & $\begin{array}{c}\text { Physician } \\
\text { Charges (\$) }\end{array}$ & $\begin{array}{c}\text { Total } \\
\text { Charges (\$) }\end{array}$ \\
\hline 1 & 38 & 1 & 10 & 35,437 & 16,280 & 51,717 \\
\hline 2 & 23 & 1 & 6 & 30,815 & 14,585 & 45,400 \\
\hline 3 & 42 & 1 & 7 & 28,668 & 15,308 & 43,976 \\
\hline 4 & 60 & 1 & 8 & 34,977 & 16,898 & 51,875 \\
\hline 5 & 39 & 1 & 8 & 33,931 & 10,937 & 44,868 \\
\hline 6 & 15 & 1 & 8 & 33,470 & 15,945 & 49,415 \\
\hline 7 & 28 & 1 & 13 & 41,163 & 17,586 & 58,749 \\
\hline 8 & 28 & 2 & 11 & 36,053 & 15,774 & 51,827 \\
\hline 9 & 28 & 1 & 8 & 32,441 & 15,616 & 48,057 \\
\hline 10 & 29 & 2 & 10 & 45,218 & 15,633 & 60,851 \\
\hline 11 & 30 & 2 & 6 & 33,590 & 14,574 & 48,164 \\
\hline 12 & 47 & 1 & 10 & 46,893 & 17,647 & 64,540 \\
\hline 13 & 23 & 1 & 6 & 33,781 & 14,681 & 48,462 \\
\hline 14 & 28 & 1 & 15 & 36,068 & 10,319 & 46,387 \\
\hline 15 & 22 & 1 & 6 & 30,031 & 15,286 & 45,317 \\
\hline 16 & 15 & 1 & 7 & 28,945 & 15,178 & 44,123 \\
\hline 17 & 41 & 2 & 11 & 62,111 & 22,625 & 84,736 \\
\hline 18 & 33 & 1 & 8 & 38,324 & 14,443 & 52,767 \\
\hline 19 & 22 & 1 & 10 & 33,839 & 13,931 & 47,770 \\
\hline 20 & 20 & 2 & 21 & 72,636 & 24,892 & 97,528 \\
\hline 21 & 41 & 1 & 11 & 35,659 & 9,832 & 45,491 \\
\hline 22 & 36 & 1 & 8 & 40,844 & 15,099 & 55,943 \\
\hline 23 & 17 & 1 & 12 & 37,203 & 15,685 & 52,888 \\
\hline 24 & 23 & 1 & 7 & 28,892 & 14,477 & 43,369 \\
\hline 25 & 13 & 1 & 8 & 31,714 & 15,700 & 47,414 \\
\hline Mean $\pm S D$ & $30 \pm 11$ & & $9 \pm 4$ & $37,708 \pm 10,179$ & $15,557 \pm 3,149$ & $53,265 \pm 12,755$ \\
\hline
\end{tabular}

and physician fees was obtained from the University of Michigan Medical Center budget and medical service plan offices, respectively. The annual increase in hospital charges was $7.4 \%$ for fiscal year 1989/1990 and $13.1 \%$ for fiscal year $1990 / 1991$. The annual increase in physician fees was $7.36 \%$ for fiscal year 1989/1990 and $5.7 \%$ for fiscal year 1990/1991. The annual physician fee increase is based on the Physicians Services Index of the Consumer Price Index using the 3-month period of February through April. The dollar values presented in this study represent the mean total of what was billed to each patient for either radiofrequency or surgical ablation. The cost to charge ratio expresses the actual cost of delivering a service as a proportion of what is billed to the patient for providing that service. In the present study, the cost-to-charge ratio for hospital services was 0.56 in the radiofrequency group and 0.61 in the surgical group. Cost-to-charge ratios are not available for professional fees.

Statistical analysis: The costs of radiofrequency and surgical ablation were compared using the unpaired $t$ test. A p value $<0.05$ was considered significant and all numerical data are expressed as mean \pm standard deviation.

\section{RESULTS}

Patient characteristics: The patients in the radiofrequency group were significantly older than the patients in the surgical group $(40 \pm 16$ vs $30 \pm 11$ years; $p$
$<0.01)$. The duration of arrhythmia symptoms before ablation was significantly longer in the radiofrequency group than in the surgical group ( $21 \pm 17$ vs $11 \pm 11$ years; $p<0.05$ ). There was no significant difference in the mean number of antiarrhythmic drugs used to treat patients before they underwent ablation $(1.9 \pm 1.4$ in the radiofrequency group versus $2.4 \pm 1.6$ in the surgical group).

Efficacy of ablation: Radiofrequency ablation was achieved successfully in 22 of 25 patients $(88 \%)$ during a single hospital admission and in 2 of 25 patients (8\%) during 2 hospital admissions. Surgery was successful for 24 of 25 patients (96\%) and 21 of 25 patients had a single hospital admission. Among the 4 patients who had $>1$ hospital admission, 3 patients required 2 hospital admissions and 1 patient had 3 hospital admissions. This latter patient (no. 12, Table I) was referred for management of refractory supraventricular tachycardia and had a 1-day hospital admission for a baseline electrophysiologic study and stabilization on a medical regimen pending surgery. He had a 7-day admission for surgical ablation of a concealed left-sided accessory pathway and a 2-day readmission 9 days after discharge from the hospital for management of atrial fibrillation.

Cost data (Tables I and II): The mean total cost of definitive therapy in the radiofrequency group was $\$ 14,919 \pm \$ 6,740$ compared with $\$ 53,265 \pm \$ 12,755$ in the surgical group $(\mathrm{p}<0.0001)$. Analysis of the 


\begin{tabular}{|c|c|c|c|c|c|c|}
\hline Pt. No. & Age (yr) & No. of APs & $\begin{array}{l}\text { Hospital } \\
\text { Stay (days) }\end{array}$ & $\begin{array}{c}\text { Hospital } \\
\text { Charges (\$) }\end{array}$ & $\begin{array}{l}\text { Physician } \\
\text { Charges (\$) }\end{array}$ & $\begin{array}{c}\text { Total } \\
\text { Charges (\$) }\end{array}$ \\
\hline 1 & 25 & 1 & 2 & 4,961 & 4,440 & 9,401 \\
\hline 2 & 31 & 1 & 4 & 9,231 & 8,380 & 17,611 \\
\hline 3 & 38 & 1 & 2 & 5,317 & 4,702 & 10,019 \\
\hline 4 & 50 & 1 & 5 & 7,502 & 4,974 & 12,476 \\
\hline 5 & 31 & 1 & 2 & 2,597 & 5,351 & 7,948 \\
\hline 6 & 40 & 1 & 5 & 7,502 & 5,243 & 12,736 \\
\hline 7 & 35 & 1 & 4 & 8,997 & 7,181 & 16,178 \\
\hline 8 & 14 & 2 & 5 & 15,149 & 16,537 & 31,686 \\
\hline 9 & 50 & 1 & 2 & 6,982 & 9,491 & 16,473 \\
\hline 10 & 24 & 1 & 2 & 7,212 & 8,904 & 16,116 \\
\hline 11 & 42 & 1 & 2 & 4,756 & 5,160 & 9,916 \\
\hline 12 & 57 & 1 & 4 & 9,174 & 8,711 & 17,885 \\
\hline 13 & 32 & 1 & 5 & 16,592 & 15,387 & 31,979 \\
\hline 14 & 42 & 1 & 3 & 6,784 & 4,203 & 10,987 \\
\hline 15 & 29 & 1 & 2 & 5,313 & 4,393 & 9,706 \\
\hline 16 & 54 & 1 & 2 & 5,619 & 5,018 & 10,637 \\
\hline 17 & 37 & 1 & 2 & 4,855 & 4,767 & 9,622 \\
\hline 18 & 16 & 1 & 2 & 4,984 & 4,242 & 9,226 \\
\hline 19 & 63 & 1 & 5 & 12,453 & 9,335 & 21,788 \\
\hline 20 & 60 & 2 & 4 & 13,090 & 12,684 & 25,774 \\
\hline 21 & 67 & 1 & 2 & 8,025 & 7,717 & 15,742 \\
\hline 22 & 34 & 1 & 2 & 5,225 & 5,087 & 10,312 \\
\hline 23 & 16 & 1 & 3 & 6,070 & 4,394 & 10,464 \\
\hline 24 & 74 & 1 & 5 & 10,282 & 7,638 & 17,920 \\
\hline 25 & 52 & 1 & 2 & 5,160 & 5,222 & 10,382 \\
\hline Mean $\pm S D$ & $40 \pm 16$ & & $3 \pm 1$ & $7,753 \pm 3,472$ & $7,166 \pm 3,439$ & $14,919 \pm 6,740$ \\
\hline
\end{tabular}

component charges in each group revealed that the mean hospital charge per patient in the radiofrequency group was $\$ 7,753 \pm \$ 3,472$ compared with $\$ 37,708 \pm$ $\$ 10,179$ in the surgical group ( $p<0.0001$ ). The mean physician charge per patient in the radiofrequency group was $\$ 7,166 \pm \$ 3,439$ compared with $\$ 15,557 \pm$ $\$ 3,149$ in the surgical group ( $p<0.0001$ ). In the radiofrequency group, the physician charge consisted almost entirely of the electrophysiologist's fee. In the surgical group, the total physician charge included professional fees billed from electrophysiology, cardiothoracic sur- gery, anesthesiology and radiology. Although both the hospital and physician charges were significantly lower in the radiofrequency group than in the surgical group, the greater relative reduction occurred in the hospital costs. Figure 1 shows the direct relation between the duration of hospital stay and the total cost of definitive therapy in the radiofrequency and surgical ablation groups. Regression analysis revealed an R-squared value of $0.78(p<0.0001)$.

Mean number of days spent in the hospital to achieve accessory pathway ablation was $3.1 \pm 1.3$ in
FIGURE 1. Scatter diagram displaying the direct relation between total cost of definitive therapy (dollars) and duration of hospital admission (days) in patients undergoing radiofrequency and surgical ablation of accessory pathways. Regression analysis revealed an R-squared value of 0.78 (p $<0.0001$ ).

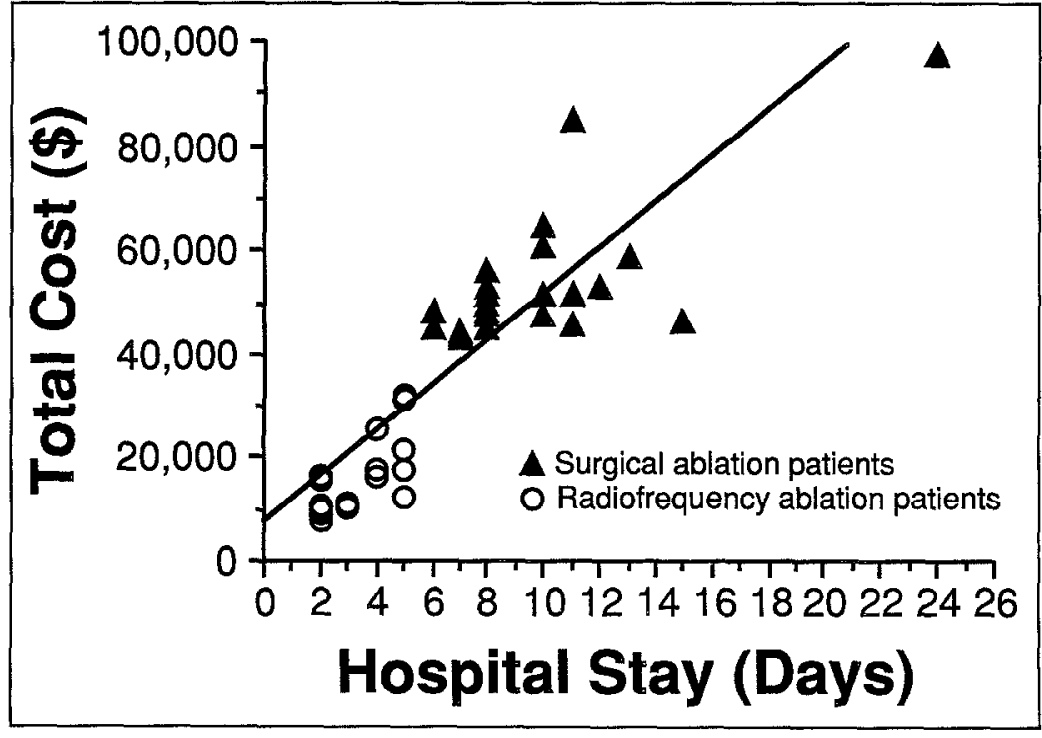

COST OF THERAPY IN WPW SYNDROME 
the radiofrequency group compared with $9.5 \pm 3.8$ in the surgical group $(p<0.0001)$. Mean number of hospitalizations deemed related to accessory pathway ablation was $1.1 \pm 0.3$ in the radiofrequency group and 1.2 \pm 0.5 in the surgical group (difference not significant). The mean time lost from work was $11 \pm 19$ days in the radiofrequency group $(n=18)$ and $55 \pm 26$ in the surgical group $(n=19)(p<0.0001)$.

Patient 20 in the surgical group had the longest admission (24 days) and the highest charges of any patient in the study, with hospital costs of $\$ 72,636$ and physician fees of $\$ 24,892$. He was a 20 -year-old white man with 2 accessory pathways (left posterolateral and posteroseptal, both manifest). After a baseline electrophysiologic test, he underwent apparently successful accessory pathway ablation. However, within hours, the delta waves reappeared on the 12-lead electrocardiogram and he was brought back to the operating room for a second attempt at surgical ablation. At a followup epicardial wire pacing study 1 week later, he was found to have persistent conduction over his posteroseptal accessory pathway and he was treated with flecainide. His postoperative recovery was prolonged due to persistent fevers.

Cost data in uncomplicated cases: Because some of the patients, particularly in the surgical group, had a more complicated course than others, a cost analysis was performed on a subset of 10 patients in each group who had a rapid and uncomplicated postprocedure recovery (patients 5, 8, 11, 13, 15, 16, 19, 22, 24 and 25 in the surgical group, and patients $1,5,9,10,11,15$, $16,18,22$ and 25 in the radiofrequency group, Tables I and II, respectively). Total, hospital and physician charges in this subset of patients undergoing uncomplicated radiofrequency and surgical ablation, respectively, were $\$ 11,012 \pm \$ 2,886$ vs $\$ 47,726 \pm \$ 3,817$ (p $<0.0001), \$ 5,281 \pm \$ 1,266$ vs $\$ 33,162 \pm \$ 3,604$ (p $<0.0001)$ and $\$ 5,731 \pm \$ 1,872$ vs $\$ 14,564 \pm \$ 1,395$ $(\mathrm{p}<0.0001)$.

\section{DISCUSSION}

The main finding of this study is that calheter-mediated radiofrequency ablation accomplishes accessory pathway interruption at a fraction of the cost of surgical ablation. This dramatic reduction in cost is due both to a reduction in hospital costs and physician charges. In the present study, the total cost of therapy in the radiofrequency group was just $28 \%$, hospital costs were $21 \%$ and physician fees were $46 \%$ of those in the surgical group. Although the most significant factor resulting in lower hospital costs in the radiofrequency group was the shorter hospital stay, other important procedure-related factors included the lack of need for operating room facilities, care in the intensive therapy unit or paramedical ancillary services such as physical therapy and respiratory care. An additional cost-limiting feature of radiofrequency energy is that accessory pathway ablation was achieved during the diagnostic electrophysiologic study in most patients. The lower physician charges in patients undergoing radiofrequency $a b$ lation reflect the absence of surgical, anesthesiology and radiology professional fees. When the cost analysis was repeated on a subgroup of patients with uncomplicated procedures, there was still a highly significant difference between the cost of radiofrequency and surgical ablation. Also, the cost-to-charge ratio for hospital services was similar in the 2 groups.

Reduced duration of hospitalization: An important finding of the present study is that radiofrequency ablation results in a marked curtailment in hospital stay. Thus, mean duration of hospital admission was 3 days in the radiofrequency group compared with 9 days in the surgical group. Almost all the patients in the radiofrequency group were walking without difficulty within 12 hours of the procedure, and the only reason for continuing their hospitalization was because of the investigational nature of the procedure. All but 1 of the patients in the radiofrequency group had a very benign post procedure course and could have been discharged from the hospital within 24 hours. Indeed, it is our current practice to discharge patients without complications from the hospital the day after undergoing radiofrequency ablation. In the future, it may even be feasible to perform radiofrequency ablation of accessory pathways on an out-patient basis in selected patients.

General features of radiofrequency ablation that promote cost-containment: There are a number of other general reasons why radiofrequency ablation is associated with cost-containment. The recent dramatic escalation in medical care costs has been attributed, in no small way, to the use of ever-advancing technology in medicine. ${ }^{5,6}$ However, radiofrequency ablation contrasts with this general trend in that it represents a new application of an old technology. The technology has been used for several years, for example to treat patients with trigeminal neuralgia, and it's recent application to patients with cardiac arrhythmias has been relatively incxpensivc. A comparison with coronary angioplasty is also pertinent. Initial experience with radiofrequency ablation suggests that repeat procedures are required less frequently than is the case with coronary angioplasty and that the initial treatment is usually curative rather than palliative. ${ }^{2}$ This feature serves to reduce medical care costs for patients undergoing radiofrequency ablation.

Comparison with previous studies: In a recent study, ${ }^{7}$ the costs of catheter-mediated direct-current shock ablation and surgical ablation in the Wolff-Par- 
kinson-White syndrome were compared. In that study, the mean total cost per patient, expressed in 1988 dollar values, was $\$ 14,116 \pm \$ 4,493$ for catheter ablation compared to $\$ 34,175 \pm \$ 5,434$ for surgical ablation ( $p$ $<0.0001$ ). The mean duration of hospitalization was 6 \pm 2 days in the group treated with shock ablation compared with $8 \pm 4$ days in the group treated surgically (difference not significant). Direct-current shock ablation also had a significant indirect economic advantage as a result of rapid return to work. The present results demonstrate that radiofrequency ablation maintains the cost containment associated with direct-current shock ablation ${ }^{7}$ and abbreviates still further the period of hospitalization for patients undergoing definitive therapy. It also permits rapid return to work with beneficial indirect economic effects.

Comparison of the cost of radiofrequency ablation and drug therapy: For the purposes of comparison, we examined the cost of treatment with a representative antiarrhythmic drug, propafenone, in a hypothetical patient with an accessory pathway and recurrent supraventricular tachycardia. In the case of a dose of $225 \mathrm{mg}$ three times daily and a 3-month prescription, refilled 4 times, the annual cost is $\$ 1,000$; if 1 year's supply is dispensed at once the cost is $\$ 985$. Thus, the annual cost for this drug alone is approximately $\$ 1,000$ and this does not take into consideration the cost of a diagnostic electrophysiologic test and electropharmacologic testing, visits to a physician or emergency room, lost productivity because of time away from work, the inconvenience associated with taking medication 3 times per day, and the possible adverse effects and lifestyle restrictions. Based on these figures, radiofrequency ablation would pay for itself in $\leq 15$ years, and any patient who required drugs for $>15$ years would save money with radiofrequency ablation. Thus, over the long term, particularly in younger patients, definitive therapy with radiofrequency ablation would appear to be more economical than pharmacologic therapy.

Use of radiofrequency ablation in patients unsultable for surgical ablation: An interesting finding of the present study is the significant difference in age and duration of symptoms between patients undergoing radiofrequency and surgical ablation of accessory pathways. Thus, mean age and symptom duration in the radiofrequency group was $40 \pm 16$ (range 14 to 74 ) and $21 \pm 17$ years, respectively, compared to $30 \pm 11$ (range 13 to 60 ) and $11 \pm 11$ years in the surgical group ( $\mathrm{p}<0.01$ and $<0.05$, respectively). These data reflect the fact that with radiofrequency ablation we were able to offer a curative procedure to a much broader spectrum of patients and to a group of patients that would not otherwise have been considered suitable candidates for definitive ablation therapy. In addition to their older age, patients in the radiofrequency group had a much higher prevalence of general medical diseases.

Study limitation: A possible limitation of this study is that the cost analysis was retrospective and open. This feature may have introduced a certain bias against surgery. However, a determined effort was made to overcome this potential bias as outlined in the Methods section. In addition, we repeated the analysis on a subgroup of 10 patients with uncomplicated procedures. The cost differentials evident in this subgroup analysis were essentially the same as those present in the overall analysis. Therefore, we feel that the cost data presented are a true and accurate estimate of the cost of these proccdurcs.

Acknowledgment: We acknowledge with gratitude the help provided by Wendy Henshaw and the staff of Internal Medicine Associates and the other physician associates groups at the University of Michigan Medical Center. We also received willing assistance from the staff of the patients' accounts office, the medical service plan office and the medical records department.

\section{REFERENCES}

1. Jackman WM, Wang X, Friday KJ, Roman CA, Moulton KP, Beckman KJ, McClelland JH, Twidale N, Hazlitt A, Prior MI, Margolis PD, Calame JD, Overholt ED, Lazzara R. Catheter ablation of accessory atrioventricular pathways (Wolff-Parkinson-White syndrome) by radiofrequency current. $N$ Engl $J$ Med 1991;324:1605-1611.

2. Calkins H, Sousa J, El-Atassi R, Rosenheck S, de Buitleir M, Kou WH Kadish AH, Langberg JJ, Morady F. Diagnosis and cure of the Wolff-ParkinsonWhite syndrome or paroxysmal supraventricular tachycardias during a single electrophysiologic test. $N$ Engl J Med 1991;324:1612-1618.

3. Cain ME, Cox JL. Surgical treatment of supraventricular tachyarrhythmias. In: Platia EV, ed. Management of Cardiac Arrhythmias - The Nonpharmacologic Approach. Philadelphia: J.B. Lippincott, 1987;304-339.

4. Josephson ME, Seides SF. Clinical Cardiac Electrophysiology. Techniques and Interpretations. Philadelphia: Lea \& Febiger, 1979;211-245.

5. Ginzberg E. High-tech medicinc and rising health earc costs. JAMA 1990;263:1820-1822.

6. Knoebel SB. Cardiology by the numbers and cost-containment. Am J Cardiol 1988;61:1112-1115.

7. De Buitleir M, Bove EL, Schmaltz S, Kadish AH, Morady F. Cost of catheter versus surgical ablation in the Wolff-Parkinson-White syndrome. Am J Cardiol $1990 ; 66: 189-192$. 